\title{
A Low-Cost Fluid Switch for Frequency-Reconfigurable Vivaldi Antenna
}

\author{
Cristina Borda-Fortuny, Member, IEEE, Kin-Fai Tong, Senior Member, IEEE, Allann Al-Armaghany, Member, IEEE, \\ and Kai-Kit Wong, Fellow, IEEE
}

\begin{abstract}
A fluid switch is proposed for a frequency-agile Vivaldi antenna whose operating frequency band can be switched between two selected bands. A study of various ionized solutions of different concentrations is performed. A 2 mol $\mathrm{KCl}$ solution is selected as the fluid for the switch because of its relatively good properties in conductivity, relative permittivity, and loss tangent. The fluid-switched reconfigurable Vivaldi antenna can function well between two user-defined operating bands: 3.2 and $4.5 \mathrm{GHz}$ with stable measured gain of $11 \mathrm{dBi}$ in both bands and an isolation of $15 \mathrm{~dB}$. This reconfigurable antenna demonstrates that a lowcost fluid switch may be an alternative device for reconfigurable antenna designs providing more flexibility.
\end{abstract}

Index Terms-Ionized solutions, reconfigurable antennas.

\section{INTRODUCTION}

$\mathbf{T}$ HE present trend to integrate several capabilities in a single wireless communication device is driving new research on frequency-reconfigurable antennas. Moreover, cognitive and software-defined radio systems demand higher antenna capabilities in terms of adaptation to changing environments. To address these new challenges, wireless communication systems are required to be cognitive and adaptable [1]. Multiband antennas are not suitable in ultra wideband systems as they cannot dynamically allocate the operating frequency bands while rejecting undesired bands for interference reduction. Frequency-reconfigurable antennas provide good out-ofband rejection while relaxing the requirements on the front-end filters or even suppressing the need for those filters [2].

Most reconfigurable antennas usually operate on a narrow frequency range, which does not preserve the required radiation characteristics over a wide spectrum [3], [4]. In this letter, a coplanar Vivaldi antenna is taken as the basis for the proposed reconfigurable antenna. Since it is a traveling-wave wideband antenna, it can maintain its radiation characteristics in a wide frequency range. The challenge is to reconfigure the Vivaldi

Manuscript received August 8, 2017; revised September 11, 2017; accepted September 26, 2017. Date of publication November 13, 2017; date of current version December 11, 2017. (Corresponding author: Kin-Fai Tong.)

The authors are with the Department of Electronic and Electrical Engineering, University College London, London WC1E 6BT, U.K. (e-mail: cristina.borda.12@ucl.ac.uk; k.tong@ucl.ac.uk; a.al-armaghany@ucl.ac.uk; kai-kit.wong@ucl.ac.uk).

Color versions of one or more of the figures in this letter are available online at http://ieeexplore.ieee.org.

Digital Object Identifier 10.1109/LAWP.2017.2759580 antennas to operate in the desirable operating frequency bands on demand.

Furthermore, frequency-reconfigurable antennas using RF switches can only provide limited capability in frequency tuning. Some of the RF switches are lossy, and the biasing circuits could be complicated and affect antenna performance significantly [5]. High-performance RF switches that can operate in the $\mathrm{GHz}$ frequency ranges could be expensive [6]. Other lowperforming RF switches, such as [7], have lower cost but present soldering challenges and introduce higher insertion losses. In this letter, an alternative method is proposed by introducing a fluid switch. Besides, ionized solutions can be beneficial because of their durability, ease to conform into desired shapes, and high-power handling capability when compared to other RF switches technology [8]. The main concerns for using fluids in antennas is the risk of leakage and their high freezing points. Therefore, creative designs are required, and the use of a threedimensional (3-D) printing technology is proposed to fabricate the fluid switch for attaching it securely onto an antenna. In consideration of the freezing point of common alcohol (for example, the freezing point of ethanol is $-114.1^{\circ} \mathrm{C}$ ), the high freezing point problem of ionized water can be resolved by addition of small amount of alcohol into the ionized water, which will not change the dielectric properties of the ionized water significantly and reduce the freezing point of ionized water to operational temperature.

A micro peristaltic pump may not be the smallest and introduces some complexity to the design, but it is not comparable to the complexity introduced by the biasing lines for RF switches. The effect of the biasing lines to the antenna is much higher as they need to be close to the propagating and radiating parts, while the micropump could be located in a distance away. Moreover, the coupling effects presented by ionized water in the rubber tube to the radiator are less significant compared to electrical signals.

In RF fluid research, the most popular fluid antenna systems use fluids like EGaIn [9] or Galinstan [10]. However, these materials react when in contact with many conventional metals such as $\mathrm{Al}, \mathrm{Ni}$, and $\mathrm{Pt}$ at room temperature [11]. Furthermore, these are expensive materials that are not suitable for low-cost systems. In the case of ionized water, $\mathrm{NaCl}$ was commonly used [12], [13], as it is abundantly available. In [14], $\mathrm{KCl}$ is proposed for the first time for fluid antennas achieving good matching and an efficiency of $70 \%$ at $2.75 \mathrm{GHz}$.

This letter aims to address the high design requirement of RF switches by the mean of conductive fluid components to switch the operating bands of a reconfigurable Vivaldi antenna. A reconfigurable Vivaldi antenna is taken as the basis of the design. A fluid switch is a good alternative to a conventional RF switch as it can form a contact at room temperature without the need of soldering and it can adapt to any shape while suppressing coupling when the fluid is drained. 


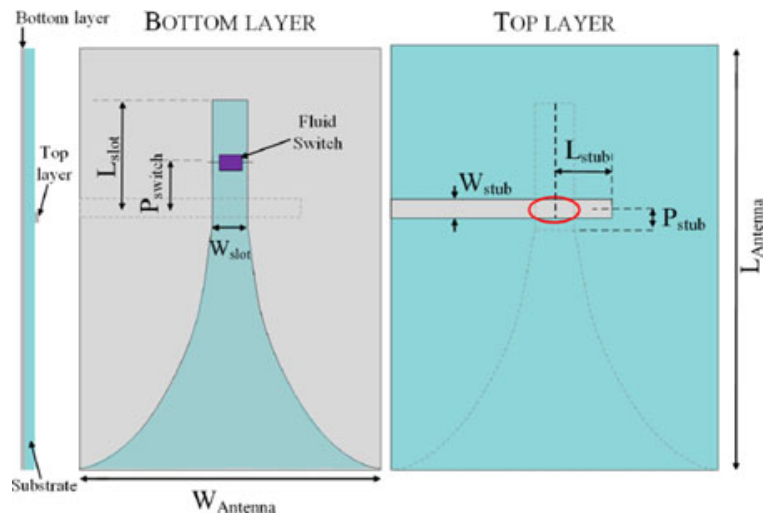

Fig. 1. Geometry of the fluid-switched design indicating design parameters.

TABLE I

DESIGN PARAMETERS FOR THE RECONFIGURABLE ANTENNA

\begin{tabular}{lccc}
\hline \hline Parameter & Value $(\mathrm{mm})$ & Parameter & Value (mm) \\
\hline $\mathrm{L}_{\text {antenna }}$ & 250.0 & $\mathrm{~W}_{\text {antenna }}$ & 150.0 \\
$\mathrm{~L}_{\text {slot }}$ & 60.0 & $\mathrm{~W}_{\text {slot }}$ & 1.22 \\
Pos $_{\text {switch }}$ & 40.0 & $\mathrm{~L}_{\text {stub }}$ & 7.98 \\
$\mathrm{~W}_{\text {stub }}$ & 1.5 & Pos $_{\text {stub }}$ & 15.8 \\
\hline \hline
\end{tabular}

\section{Antenna Geometry AND DESIGN}

The proposed antenna is designed on a single microwave substrate, as shown in Fig. 1. The design parameters are described in Table I. The dielectric substrate used is Taconic RF43 with thickness $0.762 \mathrm{~mm}$ with a relative permittivity of 4.3 . The top layer presents a microstrip feed line ending with an open-circuited stub. The microstrip line couples the signal to the back-slot. The stub-to-slot intersection is highligthed in red in the Top Layer in Fig. 1, and it is formed by two parts: microstrip open-circuited stub and the slot line. Frequency tuning is achieved by changing the effective length of the slot. This effect can be achieved by using either a physical conductive connection or a biased RF switch at the operating frequency. In this letter, a low-cost 3-D-printed case enclosing the ionized water is introduced in the slot to produce an alternative conductive path for the high-frequency band operation. The position of the fluid switch in the slot determines the effective length of the slot, thus controlling the operating frequency in high-band mode. A peristaltic pump controlled by a Raspberry Pi 2 and a motor driver was used to regulate the fluid flow in the system.

A fluid switch with $\mathrm{KCl}$ solution is introduced at the back-slot position indicated in Fig. 1. There are no biasing lines or metallic contacts near the radiating part that will generate disturbances in the antenna performance. The fluid switch is designed to be 3-D-printed using a Formlabs Form1+ printer and then bonded to the antenna at the optimized position for the RF short circuit to tune the operating frequency.

When the conductive fluid is introduced in the switch, it creates a connection at the selected point in the back-slot, which shortens the current path to produce a higher operating frequency band. To operate in the lower band, the fluid is drained from the switch through the drainage, allowing the electric surface current to use the full length of the back-slot.

A coplanar Vivaldi antenna is required to cover the whole operating frequency bands of interest, and it is designed by following the equations presented in [15].

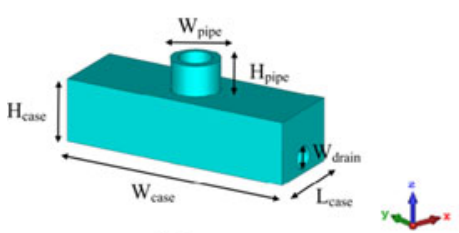

(a)

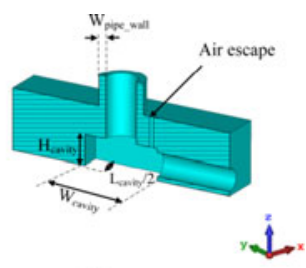

(b)
Fig. 2. (a) Case dimensions and (b) cut view of case with dimensions.

TABLE II

DESIGN PARAMETERS FOR THE CASE

\begin{tabular}{lccc}
\hline \hline Parameter & Value (mm) & Parameter & Value (mm) \\
\hline $\mathrm{L}_{\text {case }}$ & 10.0 & $\mathrm{~W}_{\text {case }}$ & 30.0 \\
$\mathrm{H}_{\text {case }}$ & 8.0 & $\mathrm{~W}_{\text {pipe }}$ & 6.2 \\
$\mathrm{H}_{\text {pipe }}$ & 4.0 & $\mathrm{~W}_{\text {pipe }}$ & 1.0 \\
$\mathrm{~L}_{\text {cavily }}$ & 5.0 & $\mathrm{~W}_{\text {cavity }}$ & 10.0 \\
$\mathrm{H}_{\text {cavity }}$ & 4.0 & $\mathrm{~W}_{\text {drain }}$ & 5.0 \\
\hline \hline
\end{tabular}

TABLE III

CONCENTRATION OF GRAMS PER Liter For EACH MEASURED Fluid

\begin{tabular}{lcccc}
\hline \hline & $0.1 \mathrm{~mol}$ & $0.5 \mathrm{~mol}$ & $1 \mathrm{~mol}$ & $2 \mathrm{~mol}$ \\
\hline $\mathrm{NaCl}$ & $5.844 \mathrm{~g}$ & $29.221 \mathrm{~g}$ & $58.443 \mathrm{~g}$ & $116.886 \mathrm{~g}$ \\
$\mathrm{KCl}$ & $7.455 \mathrm{~g}$ & $37.276 \mathrm{~g}$ & $74.551 \mathrm{~g}$ & $149.103 \mathrm{~g}$ \\
\hline \hline
\end{tabular}

Fig. 2 shows the fluid switch created to enclose the ionized water in a cavity for direct contact with the surface of the backslot in the reconfigurable Vivaldi antenna. The fluid switch is created in CST Microwave Studio introducing the measured permittivity characteristics of the resin used for 3-D printing for higher accuracy of the simulated results. The dimensions of the switch are presented in Table II.

\section{EXPERIMENT TO MEASURE IONIZED SOLUTIONS AND CONCENTRATIONS}

To determine the best ionized solution for our purposes, an experiment was set up to measure the dielectric properties of the ionized solutions of different concentrations using an Agilent 85070E Dielectric Probe Kit. $\mathrm{NaCl}$ and $\mathrm{KCl}$ provide some of the ions most responsible for salinity in seawater [16]. They are chosen because they are nontoxic, noncorrosive, and soluble in water, and they can be more conductive when compared to pure water.

The characteristics of eight different solutions of various $\mathrm{NaCl}$ and $\mathrm{KCl}$ concentrations have been measured: $0.1,0.5$, and $2 \mathrm{~mol}$ of $\mathrm{NaCl}$ and $\mathrm{KCl}$ are dissolved in deionized water. The molecular weight of $\mathrm{NaCl}$ and $\mathrm{KCl}$ is used to obtain the required weight of $\mathrm{NaCl}$ and $\mathrm{KCl}$ needed to achieve different concentration of ionized solutions, as illustrated in Table III. Although it is well known that the conductivity of the ionized solution will vary with the temperature, the aim of this letter is to demonstrate the potential of ionized fluid switches, therefore the ionized solutions are measured at a temperature of $20^{\circ} \mathrm{C}$. Furthermore, the permittivity and conductivity fluctuations due to temperature when operating this switch in a different regular environment can be compensated by adjusting the volume of the fluid in the container. The permittivity for different concentrations is measured from 500 to $8.5 \mathrm{GHz}$, which decreases with 


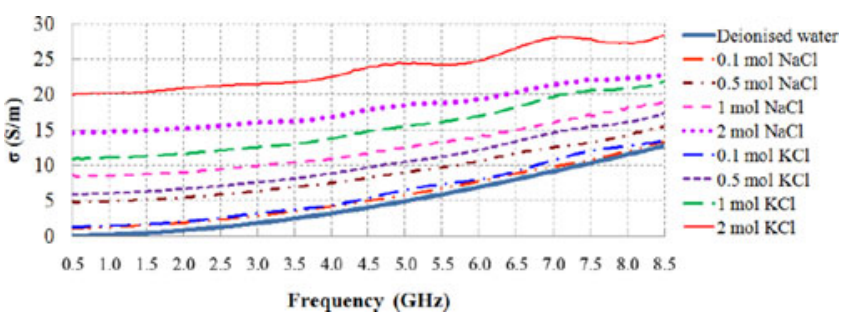

Fig. 3. Measured conductivity for different concentrations of $\mathrm{NaCl}$ and $\mathrm{KCl}$.

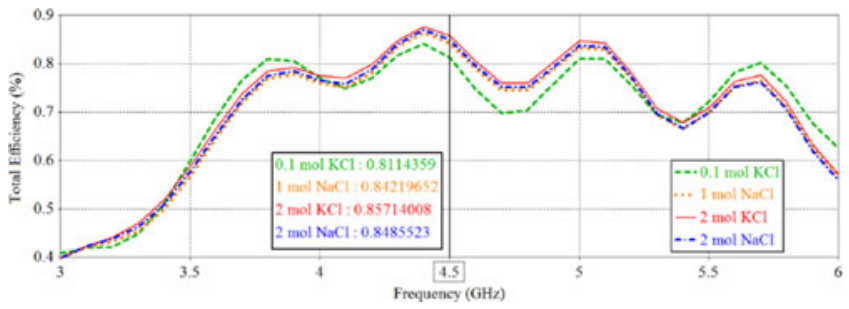

Fig. 4. Simulated total efficiency for different concentrations of $\mathrm{NaCl}$ and $\mathrm{KCl}$ in a fluid-switched reconfigurable Vivaldi antenna.

the concentration of ionized solution in water. The conductivity is extracted from the permittivity measurements following [17], as shown in Fig. 3.

The measured conductivity of ionized fluid for different concentrations is imported into a commercial full-wave electromagnetic simulation software, CST Microwave Studio, to get more accurate simulation results.

\section{ANTENNA Simulation}

\section{A. Antenna Efficiency Analysis}

In this section, the simulated efficiency of the fluid-switched Vivaldi antenna operating in the high-band mode is analyzed as the efficiency in low-band mode is less affected. Four samples of ionized water are selected because they present the maximum, minimum, and halfway between maximum and minimum conductivities shown in Fig. 3.

Fig. 4 presents the simulated total efficiency of the antenna with the four selected sampled ionized water filled in the switch. In high-band mode, the operating band starts at $3.3 \mathrm{GHz}$. All models present very low efficiency at the beginning of the band up until the operating frequency is $3.75 \mathrm{GHz}$. The highest efficiency in the operating band is provided by $2 \mathrm{~mol} \mathrm{KCl}$ solution with up to $87 \%$ and the lowest efficiency by $0.1 \mathrm{~mol} \mathrm{KCl}$ solution as expected, which is about $6 \%$ lower. The impact on the antenna efficiency caused by the conductivity of the fluid in this proposed design is very low. Therefore, the proposed ionized water switch could be used for reconfigurable antenna design with good performances. The $2 \mathrm{~mol} \mathrm{KCl}$ solution is selected for the fluid-switched Vivaldi antenna because it presents the best dielectric properties for the design.

\section{B. Analysis of Surface Current Distributions}

The current distributions for different operating bands are analyzed in this section. The operating frequency of the low band is $3.2 \mathrm{GHz}$, and the operating frequency of the high band is $4.5 \mathrm{GHz}$.

For low-band operation, the ionized water in the switch is drained, thus the two edges of the antenna at that point are not connected by the fluid switch. The low band operates at $3.2 \mathrm{GHz}$,

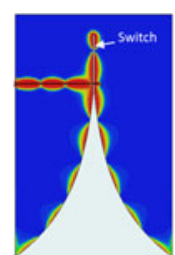

(a)

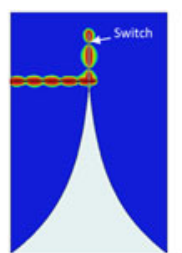

(b)

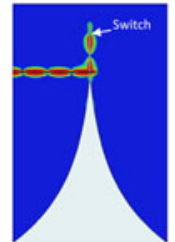

(c)

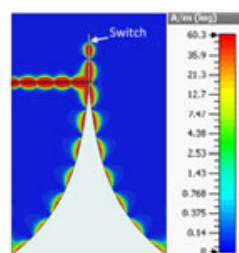

(d)
Fig. 5. Current distributions in (a) in-band low-band mode (at $3.2 \mathrm{GHz}$ ) (b) out-band low-band mode (at $4.5 \mathrm{GHz}$ ), (c) out-band high-band mode (at $3.2 \mathrm{GHz}$ ), and (d) in-band high-band mode (at $4.5 \mathrm{GHz}$ ).

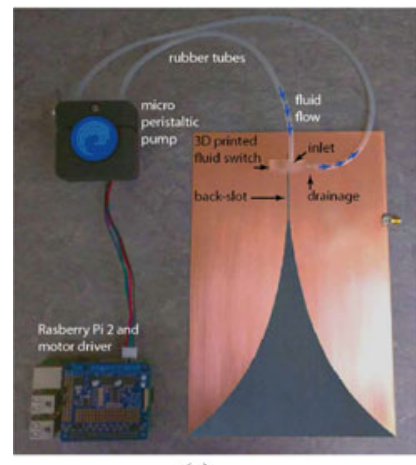

(a) (b)

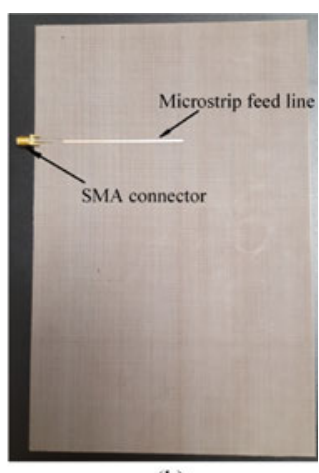

Fig. 6. (a) Bottom layer and (b) top layer of the prototype fabricated for the fluid-switched reconfigurable Vivaldi antenna.

that is, when the back-slot length is equal to $3 \lambda_{0} / 4$, as shown in Fig. 5(a). In low-band operation, at the operating frequency of $4.5 \mathrm{GHz}$, the distance from the end of the slot to the stub-to-slot intersection is calculated to be one wavelength $\left(\lambda_{0}\right)$ so that the signal is not propagating to the antenna wide end, as shown in Fig. 5(b). Such technique can improve the isolation of the two bands.

In high-band operation, the fluid is pumped in the switch, thus the back-slot presents an effective shorter length. Fig. 5(d) illustrates the current distribution at $4.5 \mathrm{GHz}$. The new effective length of the slot is $3 \lambda_{0} / 4$ at the high-band operating frequency. At $3.2 \mathrm{GHz}$, the length of the back-slot is calculated to be half-wavelength $\left(\lambda_{0} / 2\right)$, so that no signal will be coupled to the exponential flare of the antenna. Therefore, in Fig. 5(c), no signal propagates to the wide end of the Vivaldi antenna.

\section{ANTENNA VERIFICATION}

Fig. 6(a) shows the bottom layer with exponential flare of the fluid-switched reconfigurable Vivaldi antenna prototype, and Fig. 6(b) shows a picture of the top layer with the microstrip feedline. The fluid switch is located on top of the back-slot at the bottom layer. The switch was connected to a micro peristaltic pump controlled by a Rasberry Pi 2 for electronically tuning the operating frequency of the antenna.

Fig. 7(a) shows a comparison between the measured reflection coefficient (solid lines) and the simulated reflection coefficient (dashed lines). In low-band mode, the antenna performance at the higher band is affected by the fluid switch. The measured bands agree well with the simulated bands, although there are some discrepancies at higher frequencies that can be caused by the limitations of the anechoic chamber and fabrication inaccuracies.

The measured gain is compared to the simulated realized gain in Fig. 7(b). The maximum gain is stable inside the band, 


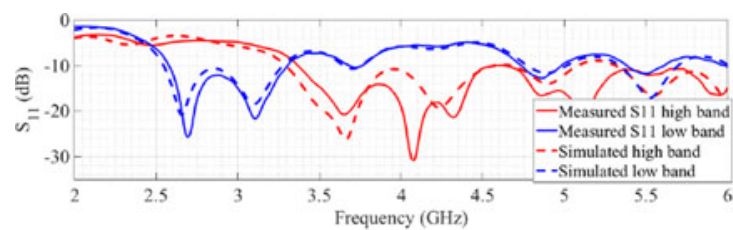

(a)

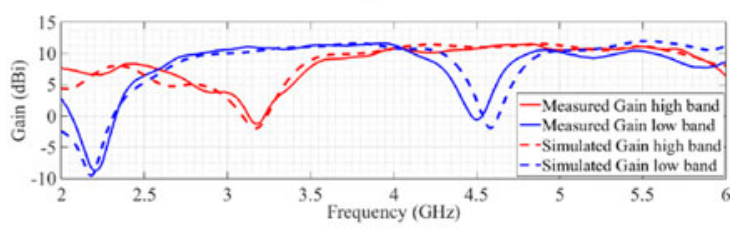

(b)

Fig. 7. Measured (solid lines) and simulated (dashed lines) for (a) $S_{11}$ and (b) gain of the fluid-switched reconfigurable Vivaldi antenna.

TABLE IV

MEASURED RESUlTS FOR THE PROPOSED DESIGN

\begin{tabular}{lccc}
\hline \hline & Center Frequency & Gain & Isolation \\
\hline Low band & $3.2 \mathrm{GHz}$ & $11 \mathrm{dBi}$ & $15 \mathrm{~dB}$ \\
High band & $4.5 \mathrm{GHz}$ & $10.9 \mathrm{dBi}$ & $14.9 \mathrm{~dB}$ \\
\hline \hline
\end{tabular}

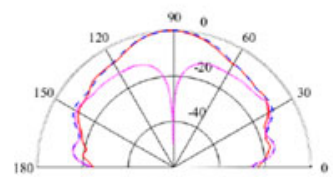

(a)

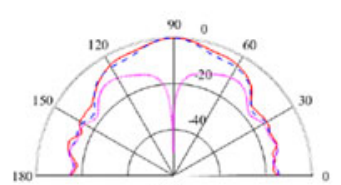

(c)

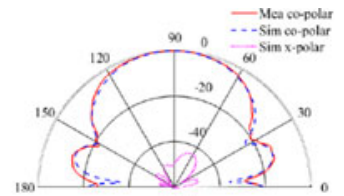

(b)

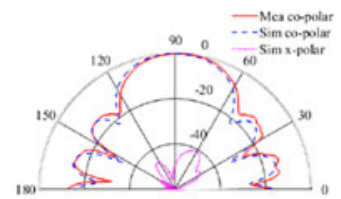

(d)
Fig. 8. Measured copolarization (red solid line), simulated copolarization (blue dashed line), and simulated cross polarization (green dashed line) (a) $E$-plane and (b) $H$-plane radiation pattern for low-band operation and (c) $E$ plane and (d) $H$-plane radiation pattern for the high-band operation of the fluid-switched reconfigurable Vivaldi antenna

and the measured rejected bands are in good agreement with the simulated bands. In low-band mode, another band appears in higher frequencies because of the introduction of the fluid switch. However, the frequencies of interest in both modes are highly isolated. The low-band mode results agree better than in high-band mode. These discrepancies are caused by fabrication inaccuracies, lossy properties of the $\mathrm{KCl}$ solution that are unaccounted in CST, and the limitations of the anechoic chamber. However, the operating bands are maintained, and high stable gain is achieved for the two bands. Table IV summarizes the characteristics of the two modes.

Fig. 8 compares the measured and simulated radiation patterns for $E$-plane [see Fig. (8a)] and $H$-plane [see Fig. 8(b)] in the low-band mode and for $E$-plane [see Fig. 8(c)] and $H$-plane [see Fig. 8(d)] in the high-band mode. The measured radiation patterns are in good agreement with the simulated ones in both modes with only a $2^{\circ}$ deviation for the high-band mode. The simulated cross-polarization patterns of the antenna are also included in figures. It is observed that similar to the common Vivaldi antennas, the $H$-plane cross-polarization level is low, about $-40 \mathrm{~dB}$, while that of the $E$ plane is relatively high, about $-15 \mathrm{~dB}$.

\section{CONCLUSION}

A low-cost reconfigurable Vivaldi antenna is proposed in this letter. The reconfiguration is achieved by introducing ionized water fluid switch on the back-slot of a Vivaldi antenna. Two mol $\mathrm{KCl}$ water solution is used as the conductive fluid, demonstrating that ionized water can be applied to reconfigurable designs in addition to those expensive materials such as EGaIn or Galinstan. Moreover, the fluid-switched Vivaldi antenna prototype presented in this letter demonstrates to be an alternative method for a conventional RF switch by a fluid switch achieving good performance under regular operating conditions, i.e., temperature above $0{ }^{\circ} \mathrm{C}$ and below boiling point. This fluid switch can be suitable in many other designs providing easy continuously frequency tuning.

\section{REFERENCES}

[1] Z. N. Chen, Handbook of Antenna Technologies. Berlin, Germany: Springer, 2015.

[2] S. Yang, C. Zhang, H. K. Pan, A. E. Fathy, and V. K. Nair, "Frequencyreconfigurable antennas for multiradio wireless platforms," IEEE Microw. Mag., vol. 10, no. 1, pp. 66-83, Feb. 2009.

[3] L. Liu and R. Langley, "Electrically small antenna tuning techniques," in Proc. Antennas Propag. Conf., 2009, pp. 313-316.

[4] J. Kiriazi, H. Ghali, H. Ragaie, and H. Haddara, "Reconfigurable dual-band dipole antenna on silicon using series MEMS switches," in Proc. IEEE Antennas Propag. Soc. Int. Symp., vol. 1, 2003, pp. 403-406.

[5] G. Wang, T. Polley, A. Hunt, and J. Papapolymerou, "A high performance tunable RF MEMS switch using barium strontium titanate (BST) dielectrics for reconfigurable antennas and phased arrays," IEEE Antennas Wireless Propag. Lett., vol. 4, pp. 217-220, 2005.

[6] Radant, "Radant MEMS," May 2015 [Online]. Available: http://www. radantmems.com/radantmems/products.html

[7] Y. Li, Z. Zhang, J. Zheng, Z. Feng, and M. F. Iskander, "Experimental analysis of a wideband pattern diversity antenna with compact reconfigurable CPW-to-slotline transition feed," IEEE Trans. Antennas Propag., vol. 59, no. 11, pp. 4222-4228, Nov. 2011.

[8] C. Murray and R. Franklin, "Independently tunable annular slot antenna resonant frequencies using fluids," IEEE Antennas Wireless Propag. Lett., vol. 13, pp. 1449-1452, 2014.

[9] G. Hayes, J. So, A. Qusba, M. Dickey, and G. Lazzi, "Flexible liquid metal alloy (EGaIn) microstrip patch antenna," IEEE Trans. Antennas Propag., vol. 60, no. 5, pp. 2151-2156, May 2012.

[10] J. So, J. Thelen, A. Qusba, G. Hayes, G. Lazzi, M. Dickey, "Reversibly deformable and mechanically tunable fluidic antennas," Adv. Funct. Mater., vol. 19, no. 22, pp. 3632-3637, 2009.

[11] P. Ahlberg et al., "Graphene as a diffusion barrier in galinstan-solid metal contacts," IEEE Trans. Electron Devices, vol. 61, no. 8, pp. 2996-3000, Aug. 2014.

[12] S. Akimoto, T. Yanagi, T. Fukasawa, and H. Miyashita, "Demonstration of a highly efficient seawater antenna," in Proc. IEEE-APS Topical Conf. Antennas Propag. Wireless Commun., Sep. 2016, pp. 55-58.

[13] C. Feng, F. P. Deng, L. Zhou, G. Hong, and W. X. Zhang, "Research on the radiation characteristics for the seawater antenna," in Proc. 2016 IEEE Int. Conf. Microw. Millim. Wave Technol, 2016, vol. 2, pp. 826-828.

[14] K. F. Tong, C. Borda-Fortuny, and J. Bai, "Low cost 3D-printed monopole fluid antenna," in Proc. Int. Symp. Antennas Propag., Nov. 2015, pp. 1-4.

[15] A. A. H. Ameri, G. Kompa, and A. Bangert, "Study about tem horn size reduction for ultrawideband radar application," in Proc. German Microw. Conf., Mar. 2011, pp. 1-4.

[16] A. G. Dickson and C. Goyet, Handbook of Methods for the Analysis of the Various Parameters of the Carbon Dioxide System in Sea Water. Oak Ridge, TN, USA: DOE, 1994.

[17] C. A. Balanis, Antenna Theory: Analysis and Design. New York, NY, USA: Wiley, 2016. 